\title{
Experimental characterization of the interaction zone between counter- propagating Taylor Sedov blast waves
}

Cite as: Phys. Plasmas 27, 022111 (2020); https://doi.org/10.1063/1.5137795

Submitted: 13 November 2019 . Accepted: 12 January 2020 . Published Online: 13 February 2020

B. Albertazzi, (D) P. Mabey, Th. Michel, (D) G. Rigon, J.-R. Marquès, S. Pikuz, S. Ryazantsev, E. Falize, L. Van Box Som, J. Meinecke, N. Ozaki, A. Ciardi, G. Gregori, and (D) M. Koenig

\section{COLLECTIONS}

F This paper was selected as Featured

SCI This paper was selected as Scilight
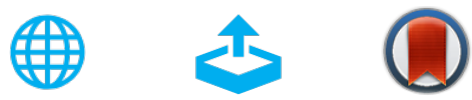

\section{ARTICLES YOU MAY BE INTERESTED IN}

Three-dimensional signatures of self-similarity in a high-energy-density plasma shear-driven mixing layer

Physics of Plasmas 27, 032701 (2020); https://doi.org/10.1063/1.5122980

Modeling magnetic confinement of laser-generated plasma in cylindrical geometry leading to disk-shaped structures

Physics of Plasmas 27, 022116 (2020); https://doi.org/10.1063/1.5139888

Scaling laws for dynamical plasma phenomena

Physics of Plasmas 25, 100501 (2018); https://doi.org/10.1063/1.5042254

\section{AlP Advances Fluids and Plasmas Collection}




\title{
Experimental characterization of the interaction zone between counter-propagating Taylor Sedov blast waves $\circledast$
}

Cite as: Phys. Plasmas 27, 022111 (2020); doi: 10.1063/1.5137795

Submitted: 13 November 2019 • Accepted: 12 January 2020 •

Published Online: 13 February 2020

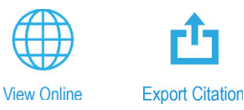

B. Albertazzi, ${ }^{1, a)}$ P. Mabey, ${ }^{7}$ (D) Th. Michel, ${ }^{7}$ G. Rigon, ${ }^{7}$ (D J.-R. Marquès, ${ }^{7}$ S. Pikuz, ${ }^{2,3}$ S. Ryazantsev, ${ }^{2,3}$ E. Falize, ${ }^{4}$ L. Van Box Som, ${ }^{4}$ J. Meinecke, ${ }^{5}$ N. Ozaki, ${ }^{6,7}$ A. Ciardi, ${ }^{8}$ G. Gregori, ${ }^{5}$ and M. Koenig ${ }^{7}$ (D)

\author{
AFFILIATIONS \\ 'LULI-CNRS, CEA, Sorbonne Universités, Ecole Polytechnique, Institut Polytechnique de Paris-F-91120 Palaiseau Cedex, France \\ 2Joint Institute for High Temperatures RAS, 13-2 Izhorskaya st., Moscow, 125412 Russia \\ ${ }^{3}$ National Research Nuclear University “MEPhl," Moscow 115409, Russia \\ ${ }^{4}$ CEA-DAM-DIF, F-91297 Arpajon, France \\ ${ }^{5}$ Clarendon Laboratory, University of Oxford, Parks Road, Oxford OX1 3PU, United Kingdom \\ ${ }^{6}$ Graduate School of Engineering, Osaka University, Suita, Osaka 565-0871, Japan \\ ${ }^{7}$ Institute of Laser Engineering, Osaka University, Suita, Osaka 565-0871, Japan \\ ${ }^{8}$ Sorbonne Université, Observatoire de Paris, Université PSL, CNRS, LERMA, F-75005, Paris, France \\ a)b.albertazzi@hotmail.fr
}

\begin{abstract}
Astronomical observations reveal that the interaction between shock waves and/or blast waves with astrophysical objects (molecular clouds, stars, jet winds, etc.) is a common process which leads to a more intricate structure of the interstellar medium. In particular, when two isolated massive stars are relatively close and explode, the resulting Supernovae Remnants (SNRs) can interact. The impact zone presents fascinating complex hydrodynamic physics which depends on the age of the SNRs, their relative evolution stage, and the distance between the two stars. In this Letter, we investigate experimentally the interaction region (IR) formed when two blast waves (BWs) collide during their Taylor-Sedov expansion phase. The two BWs are produced by the laser irradiation ( $1 \mathrm{~ns}, \sim 500 \mathrm{~J})$ of $300 \mu \mathrm{m}$ diameter carbon rods and propagate in different gases $\left(\mathrm{Ar}\right.$ and $\mathrm{N}_{2}$ ) at different pressures. The physical parameters, such as the density and temperature of the IR, are measured for the first time using a set of optical diagnostics (interferometry, schlieren, time-resolved optical spectroscopy, etc.). This allows us to determine precisely the thermodynamic conditions of the IR. A compression ratio of $r \sim 1.75$ is found and a $17-20 \%$ increase in temperature is measured compared to the shell of a single blast wave. Moreover, we observe the generation of vorticity, inducing strong electron density gradients, in the IR at long periods after the interaction. This could in principle generate magnetic fields through the Biermann Battery effect.
\end{abstract}

Published under license by AIP Publishing. https://doi.org/10.1063/1.5137795

\section{INTRODUCTION}

The physics of supernova remnants (SNRs) interacting with the ambient medium is fundamental in the structure and the dynamics of the interstellar medium (ISM) and also in high-energy astrophysics. It has been shown in Ref. 1, for example, that intersecting SNRs can lead to the generation of hot interstellar regions $\left(\mathrm{T} \sim 10^{6} \mathrm{~K}\right.$ ) and that these regions represent $\sim 30 \%$ of the volume of a spiral arm. More specifically, when two isolated massive stars are relatively close and explode, the resulting SNR can interact and lead to a more complex structure of the ISM. The impact zone presents a fascinating complex hydrodynamic physics which depends on the age of the SNR, its relative evolution stage, and the relative position of the two stars. Given the dynamical time of such systems ( $\sim 1000$ years), the time evolution of the impact region is inaccessible for observations. From the point of view of astronomical observations, the SNR candidates to collision are the ones which present a double-shell morphology such as SNRs DEM L316 ${ }^{2}$ and 3 C $400.2^{3}$ or in the Cygnus Loop. ${ }^{4}$ This conclusion is only based on multi-dimensional numerical simulations because the multiwavelength studies of emitted radiation coming from these astrophysical objects are not sufficient to constrain the origin of this specific structure. ${ }^{5,6}$ Indeed, the double-shell morphology can be induced by an external cause, mainly the inhomogeneous structure of the ISM, 
and the internal cause of the remnant as the influence of strong winds or jets emitted by the central compact object on the post-shocked medium. Since the time evolution is inaccessible, it is difficult to know if the double shell structure is due to the optical projection of the SNR or due to a real impact.

In this paper, a different approach has been used to try to improve our knowledge of the two blast wave interaction region (IR). The main objective of this study is to determine the characteristics of such a region in terms of density and temperature to help (i) benchmark numerical codes and (ii) make predictions of x-ray and optical emission to compare directly to astronomical observations. Indeed, astronomical observations of DEM L316 showed forbidden spectral lines in the interaction region between the two SNRs, which could be related to the interaction of two blast waves.

Single blast waves have been generated and studied in the frame of laboratory astrophysics for more than two decades. Indeed, scaling laws allow to make a link between the laboratory experiment and the astrophysical systems under study ${ }^{8}$ for an optically thin medium. Recent theoretical works ${ }^{9,10}$ have demonstrated that astronomical blast wave collisions present very interesting similarity properties with those generated using powerful lasers. As a consequence, a dedicated laboratory experiment has been performed allowing for the study of the interaction zone between two blast waves which collide. Two common experimental techniques have been used to study the blast wave in the laboratory. The first one focuses a high intensity laser (sub picosecond pulse duration, $I>10^{15} \mathrm{~W} \mathrm{~cm}^{-3}$ ) in clustered gas targets, ${ }^{11-18}$ while the second one uses long pulse ( $>100 \mathrm{ps}$ ), high energy ( $\gg 10 \mathrm{~J})$ laser beams focused on small diameter pins or foils. ${ }^{19-23}$ These experiments enabled us to study, for example, the stability of the shock front, i.e., the dynamics of hydrodynamical perturbations, which is of prime importance for the understanding of the Supernova's blast wave evolution. Indeed, several instabilities may develop such as Rayleigh-Taylor, Richtmeyer-Meshkov, or the Vishniac overstability. The second experimental interest of creating such a blast wave in the laboratory is to study the dynamics of the radiative blast wave, i.e., when radiation losses affect the dynamics of the blast wave. ${ }^{18}$ It has been shown that, in these conditions, a radiative precursor is present ahead of the shock front which modify the medium prior to the arrival of the shock and the deceleration takes the form $<t^{2 / 5}$, clearly different from a common Taylor-Sedov phase $\left(t^{2 / 5}\right)$. Conversely, experiments on counter-propagating plasmas and/or shocks are rare, ${ }^{24-28}$ mainly due to technical challenges and most of the study referred to a weak shock.

In this Letter, the interaction region produced when two blast waves, in their Taylor-Sedov phase collide, is characterized in terms of density and temperature conditions. The paper is organized as follows. The experimental platform is presented in Sec. II. Section III shows the single blast wave morphology and the evolution of thermodynamic parameters for different initial conditions. This will serve as a reference when comparing the physical conditions of the interaction region. Section IV shows the experimental analysis of the interaction region between the two blast waves. These data can be used to benchmark hydro/MHD codes. Finally, a discussion with implications for astrophysical systems is initiated and concludes the paper.

\section{EXPERIMENTAL SETUP}

The experiment has been performed on the LULI2000 laser facility at LULI Laboratory (Ecole Polytechnique, France). The experimental chamber is filled with different gases $\left(N_{2}, \mathrm{Ar}\right)$ at different pressures (5.7 and 11.4 mbar in the $N_{2}$ case, and 2 and 7 mbar for Ar). Two long $(\tau \sim 1 \mathrm{~ns})$, high energy $\left(E_{0} \sim 400-500 \mathrm{~J}\right.$, at the wavelength $\lambda_{L}=527 \mathrm{~nm}$, with $200 \mu \mathrm{m}$ focal spot) laser pulses irradiate two $300 \mu \mathrm{m}$ diameter carbon rods to produce two blast waves (BWs). Indeed, the short spatial and time scales of the deposited energy from the laser onto the rod produced an explosion which gives rise to the emergence of a nearly spherical blast wave propagating in the background gas. The distance between the two rods is set to $2 \mathrm{~cm}$ (cf. Fig. 1). This distance has been chosen in order for both BW's to be in their Taylor-Sedov phase when they collide, while keeping a high velocity at the beginning of the impact. Diverse optical diagnostics have been implemented perpendicular to the main laser axis. A probe laser beam (few $\mathrm{mJ}, \sim 7 \mathrm{~ns}$ at $532 \mathrm{~nm}$ ) provided some of the fundamental parameters of the experiment: (i) a $2 \mathrm{D}$ schlieren image which indicates the presence of a strong electron density gradient $\left(\nabla n_{e}\right)$ for an electron density below $n_{e}<1 \times 10^{20} \mathrm{~cm}^{-3}$, to get the $\mathrm{BW}$ radius evolution, (ii) a 2D Mach-Zehnder interferometer giving the electron density of the blast wave in the range $\sim 5 \times 10^{17}-1 \times 10^{20} \mathrm{~cm}^{-3}$ through Abel inversion, ${ }^{29}$ and (iii) time resolved optical spectroscopy in the range $\sim 450-800 \mathrm{~nm}$ to get simultaneously the electron density and temperature of the probed region using the code PrismSPECT. ${ }^{30}$ It is important to note that optical spectroscopy is code dependent and cannot resolve the shock front (the size of the probed zone is evaluated to be $\sim 400 \mu \mathrm{m}$ according to our optical system).

\section{SINGLE BLAST WAVE MORPHOLOGY AND EVOLUTION}

This section concentrates on the experimental analysis of the propagation of a single blast wave (BW) for various initial conditions (nature and pressure of the gas). Indeed, it is important to have a complete characterization of the single BW case to compare with the interaction region formed by the collision of two BWs. Its radius can be defined as

$$
R(t)=\beta t^{\alpha},
$$

where $\beta$ depends on the polytropic index $\gamma$, the initial energy $E_{0}$, and the density of the gas, and the deceleration parameter takes the form:

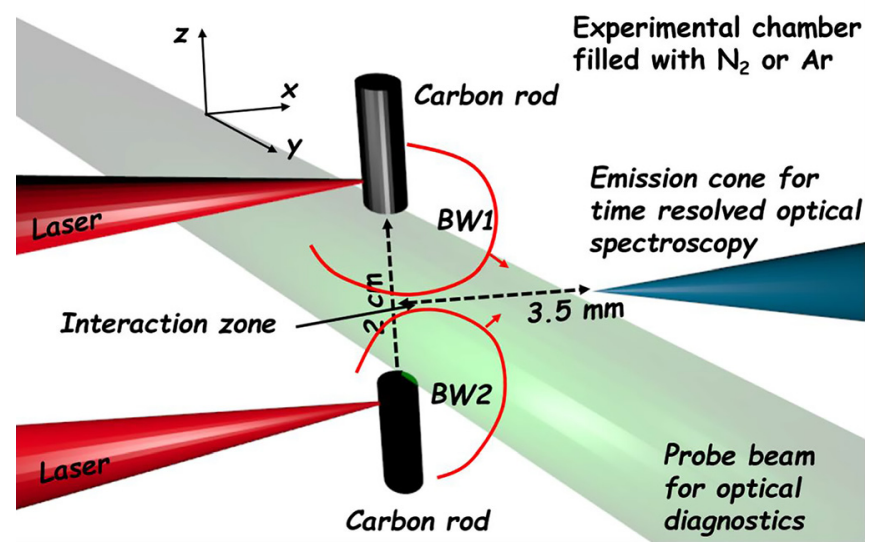

FIG. 1. Experimental setup. The whole chamber is filled with different gases $\left(N_{2}\right.$ or $\left.A r\right)$. 
$\alpha=V t / R$, where $V$ is the velocity and $R$ is the radius of the blast wave at time $t$ after the interaction (or the explosion). ${ }^{14,18}$ The $\alpha$-parameter allows to identify the evolutionary regime of the BW. For $\alpha=2 / 5$, the blast wave is in the Taylor-Sedov phase ${ }^{31}$ in spherical geometry ( $\alpha=1 / 2$ for cylindrical geometry), i.e., when the mass of the shell becomes comparable with the mass swept up by the wave. If $\alpha<2 \backslash 5$ (in spherical geometry), the BW enters in the radiative regime where energy losses through radiation cannot be neglected anymore. This radiation can heat an optically thick medium prior to the arrival of the BW and ionize it. As a consequence, an ionized precursor is formed ahead of the shock front and modifies the thermodynamic conditions. ${ }^{21}$ However, it is difficult to dissociate the preheating due to the $\mathrm{x}$-ray produced during the interaction between the carbon rod and the high power laser and the heating due to radiation. ${ }^{32}$ We will see in the following that the region probed by the time-resolved optical spectroscopy diagnostic helps to determine (i) if a precursor is present and (ii) its physical conditions $(\rho, T)$.

\section{A. Morphology, radius, and velocity}

In Figs. 2(a) and 2(c), an example of a single BW propagating in an Ar gas at different pressures as well as the determination of its radius are given. Figure 2(d), presents an example of the interaction between a single blast wave and a solid obstacle. And finally, Fig. 2(b) gives an example of two single BW's propagating toward each other. As can be seen, the BW's are not perfectly symmetrical but exhibit a clear asymmetry. Several explanations can be attributed. First, the propagation of the laser in the background gas can induce its heating and consequently its ionization. This modifies the initial conditions of the propagation of the BW and give rise to a non-spherical BW. The second is the presence of the target holder (bottom of the BW for all images) which disturbs the conditions of propagation of the BW. Figure 2(e) represents the radius of the BW determined by either schlieren and interferometry data. As one can see, it is possible to fit the radius of the BW as $R(t)=\beta t^{\alpha}$ with $\alpha=0.4 \pm 0.03$, independent of the nature or pressure of the gas. This indicates that they are in the Taylor-Sedov regime as expected. This is an important point for comparison with astronomical systems such as DEM L316, as both SNRs are still in their Taylor-Sedov phase. ${ }^{2}$ The typical velocities are summarized in Fig. 2(f). The collision time at a low gas density pressure (Ar 2 mbar and $N_{2} 5.7$ mbar) is about $150 \mathrm{~ns}$ after the beginning of the interaction. This corresponds to a velocity of about $70 \mathrm{~km} / \mathrm{s}$. At higher gas pressure, it is of the order of $350 \mathrm{~ns}$ and a corresponding velocity of $\sim 30 \mathrm{~km} / \mathrm{s}$. In the following, we will focus our attention on $N_{2}$ gas as it is easier for numerical perspectives.

\section{B. Density and temperature}

In order to evaluate the density and temperature of the blast wave and to determine the difference with the interaction region produced when two BWs collide, two diagnostics have been used: interferometry and time resolved optical spectroscopy. It is important to note that the electron density retrieved from interferometric data suffers from an important caveat. Indeed, the system exhibits a spherical symmetry rather than a cylindrical symmetry one, which is necessary for Abel inversion. The analysis has been done assuming a cylindrical symmetry but uncertainties are substantial (evaluated to be $>20 \%$ ).
As can be seen in Fig. 3, the electron density for the blast wave in the case of 2 mbar Ar and 5.7 mbar $N_{2}$ is about $\sim 1 \times 10^{19} \mathrm{~cm}^{-3}$, while for the $7 \mathrm{mbar}$ Ar and $11.4 \mathrm{mbar} N_{2}$, it is $\sim 2 \times 10^{19} \mathrm{~cm}^{-3}$. The post shock region exhibits an electron density from 5 to $10 \times 10^{18} \mathrm{~cm}^{-3}$. The thickness of the shock front is of the order of few hundreds of micrometers. These data help to constraint the numerical simulation PrismSPECT for the interpretation of time-resolved optical spectroscopy.

The results of time-resolved optical spectroscopy have been analyzed using the code PrismSPECT which is a collisional-radiative spectral analysis code designed to simulate the atomic and radiative properties of plasmas. In these simulations, we assume a plasma at a local thermodynamical equilibrium (LTE) using a Maxwellian electron distribution. Simulations have been performed for spatially uniform plasmas where free parameters are the (i) electron density and (ii) temperature. However, the electron density has been constraint by interferometric data. It is important to note that the volume probed by the diagnostic is large ( $\sim 400 \mu \mathrm{m}$ in length according to our optical system) and so it is difficult to clearly resolve the shock front. Moreover, values given are code dependent and might vary according to the model used. Here, we used the all levels model as we are not limited by computational time. Some other models have been tested (Emission Visible/UV/EUV Spectroscopy and PROPACEOS) but remain less precise than the models that include all the levels, as, by definition, they omit some lines which are seen experimentally. The spectral range is from $\sim 450 \mathrm{~nm}$ to $750 \mathrm{~nm}$. The spectral and spatial responses of the streak camera have been taken into account. The spectral resolution of the diagnostics has been evaluated to be of $\sim 1 \mathrm{~nm}$. A typical image for $N_{2}$ at 5.7 mbar is displayed in Fig. 4. The analysis of such experimental data brings us a wealth of information: (i) the shock front reaches the probed zone (located at $3.5 \mathrm{~mm}$ in lateral and $1 \mathrm{~cm}$ on the top, see Fig. 1) $200 \mathrm{~ns}$ after the laser irradiation. (ii) The progressive emergence of lines (see at $501.6 \mathrm{~nm}$ for example) prior to the arrival of the shock front indicates the presence of gas heating due to $\mathrm{x}$-ray. These $\mathrm{x}$-rays can be produced during the laser-matter interaction or in the shock front. This has strong implications as it modifies the thermodynamics conditions of the optically thick medium and as a consequence the propagation of the BW. (iii) The ratio between lines varies during time implying different $(\rho, T)$ conditions. These difference allow us to get the time evolution of $(\rho, T)$.

\section{Ionization prior to the arrival of the shock front}

As can be seen in Fig. 5(a), spectral lines appear prior to the arrival of the shock front (between $\sim 80$ and $200 \mathrm{~ns}$ ) which indicates the presence of an ionizing precursor at $\mathrm{t} \sim 80 \mathrm{~ns}$ after the beginning of the interaction. We will concentrate our analysis on two lines: around $500(501.6 \mathrm{~nm})$ and $570 \mathrm{~nm}(569.9 \mathrm{~nm})$. The comparison between the PrismSPECT simulation and experimental data is shown in Fig. 5(b). The best fit is used for $\rho=6.67 \times 10^{-6} \mathrm{~g} \mathrm{~cm}^{-3}$ (corresponding to the initial gas density) and $T=1.86 \pm 0.20 \mathrm{eV}$. The presence of a precursor has important consequences when developing scaling laws for comparison with astrophysical systems. Indeed, its parameters are modified according to the propagation medium.

\section{Determination of the physical conditions of the blast wave}

The main objective of this section is to fully characterize the density and temperature profiles of a single BW through analysis of 
Ar 2 mbar

(a)

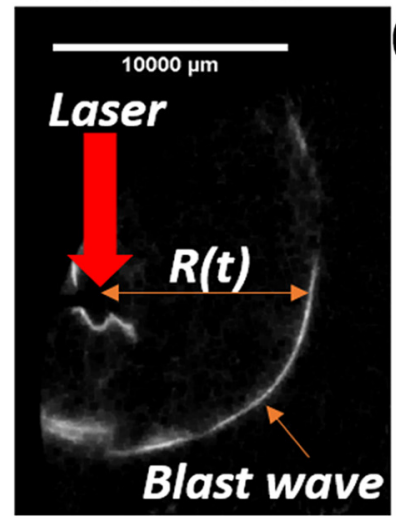

$t=100 \mathrm{~ns}$

(c)

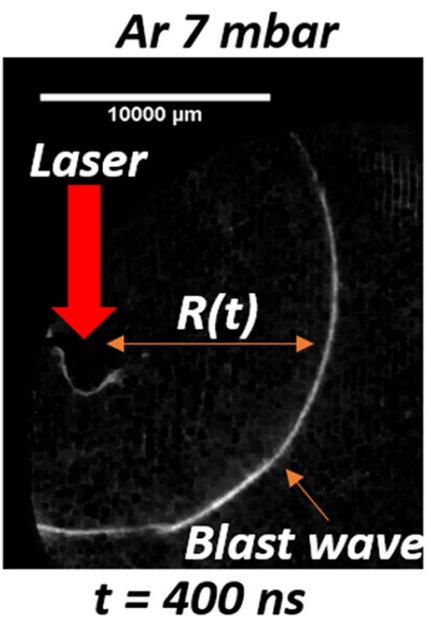

$N_{2} 5.7$ mbar

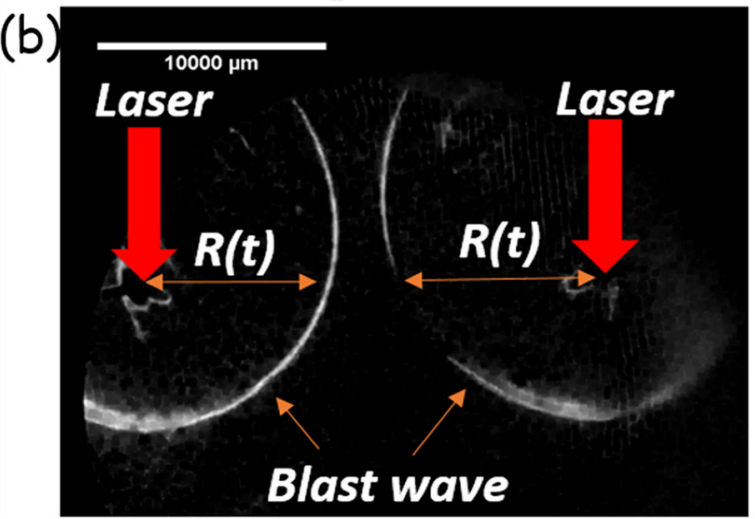

$t=100$ ns

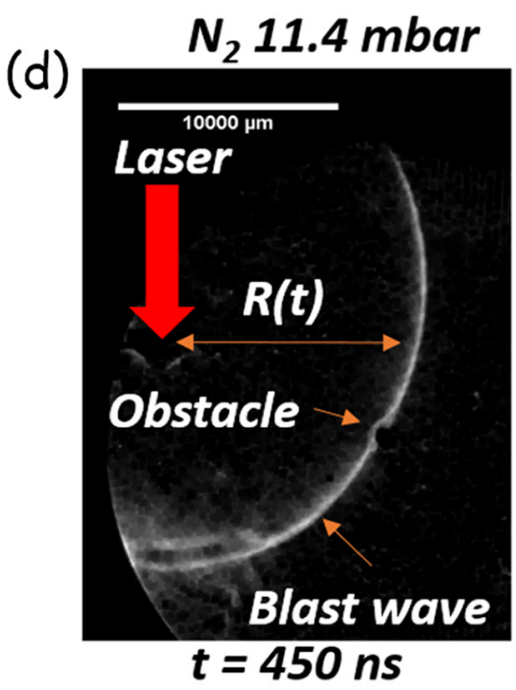

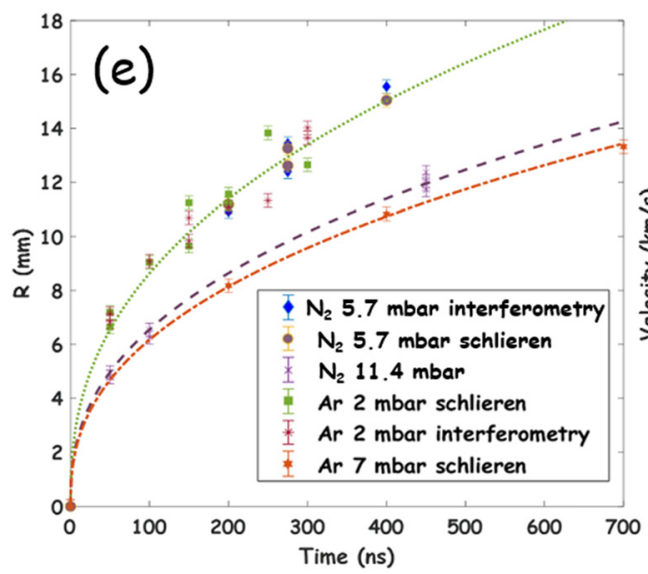

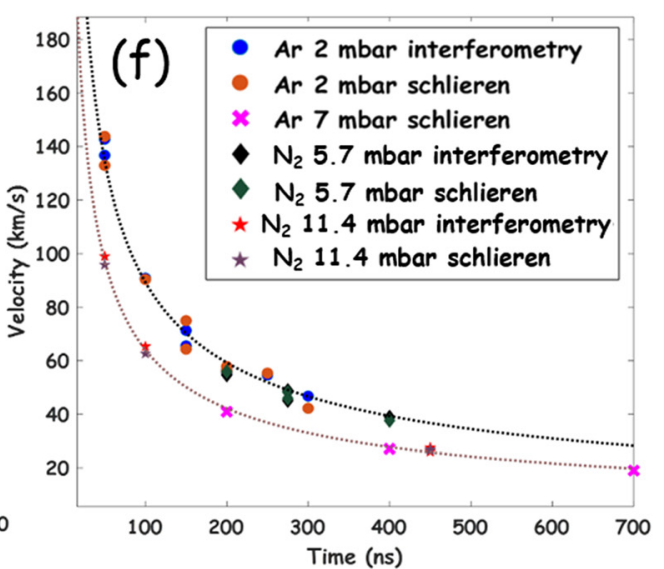

FIG. 2. Typical schlieren data of a single blast wave propagating (a) in 2 mbar Ar gas, (c) in 7 mbar Ar, and (d) in $11.4 \mathrm{mbar} \mathrm{N}_{2}$ where a spherical obstacle in present. (b) Typical example of two blast waves propagating toward each other in 5.7 mbar $N_{2}$. (e) Distance traveled by the blast wave [radius $\mathrm{R}$ taken as illustrated in (a)-(d)]. All dashed lines in (e) used the form $R(t)=\beta t^{\alpha}$ with $\alpha=0.4 \pm 0.03$. (f) Shock velocity vs time for different initial conditions of gas and pressure. 


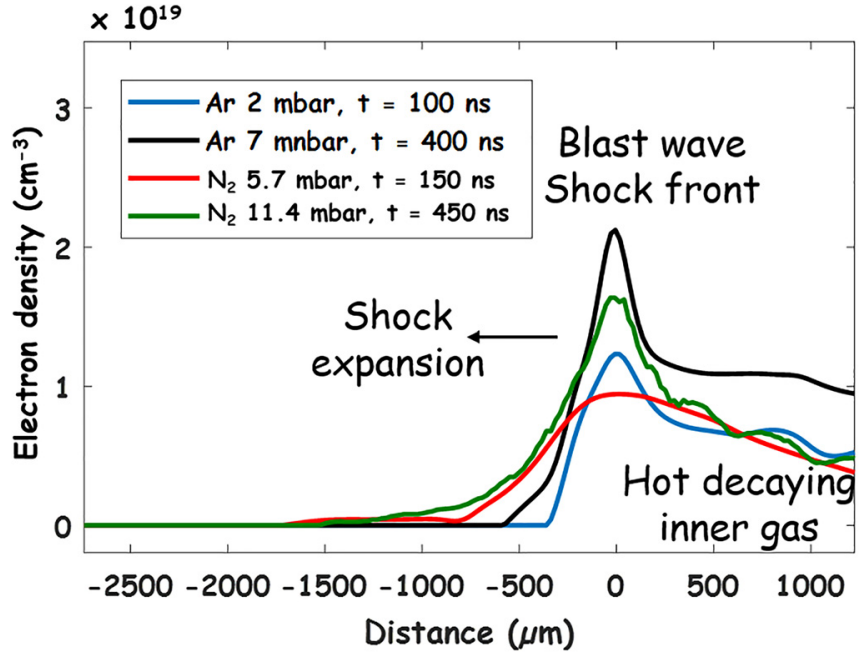

FIG. 3. Electron density retrieved from interferometric data through Abel inversion for different gases, pressures, and times.

time-resolved optical spectroscopy to compare to the IR formed by the collision of two blast waves. The density is the most difficult parameter to determine as spectral lines are not sensitive to a small variation. To fulfill this objective, PrismSPECT simulations have been performed at a fixed temperature $(T=3.9 \mathrm{eV})$, which corresponds approximately to the BW temperature (determined by preliminary simulations), and by varying the density. The full width half maximum (FWHM) of the $501.6 \mathrm{~nm}$ lines is then evaluated and directly compared to the experimental data [see Fig. 6(a)]. The spectral resolution of the diagnostic $(1 \mathrm{~nm})$ has been taken into account.

Figure 6(b) displays the $501.6 \mathrm{~nm}$ line observed experimentally at different times (see Fig. 5) and allows to deduce its FWHM. At an early time $(\mathrm{t}=234 \mathrm{~ns})$, the FWHM is $\sim 2.8 \mathrm{~nm}$, corresponding to a density of $\sim 3.10^{-5}$ g. $\mathrm{cm}^{-3}$. The width of the peak does not evolve significantly

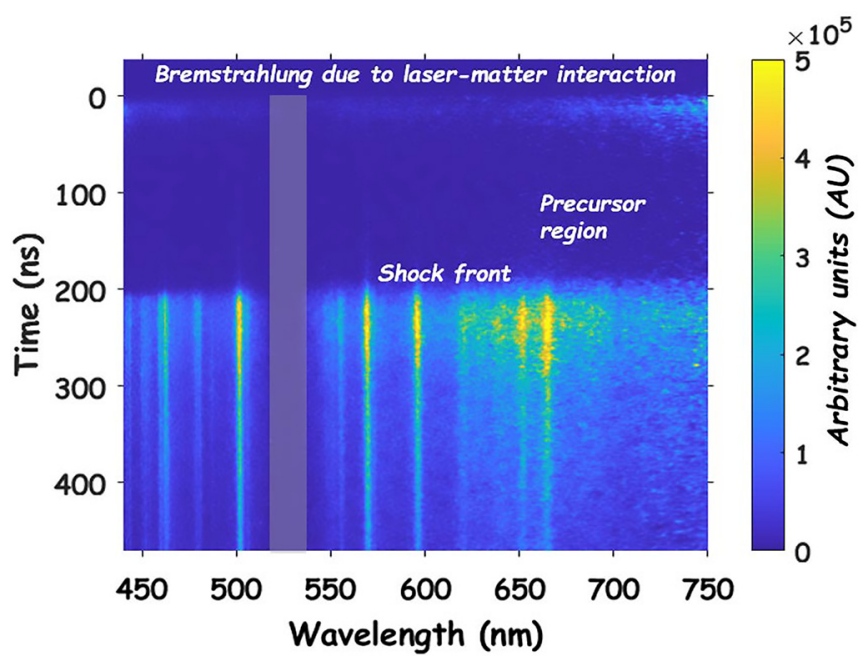

FIG. 4. Time resolved optical spectroscopy data with one blast wave propagating in $5.7 \mathrm{mbar}$ of $\mathrm{N}_{2}$. A notch filter (gray band) blocks the laser line $(526 \mathrm{~nm})$.

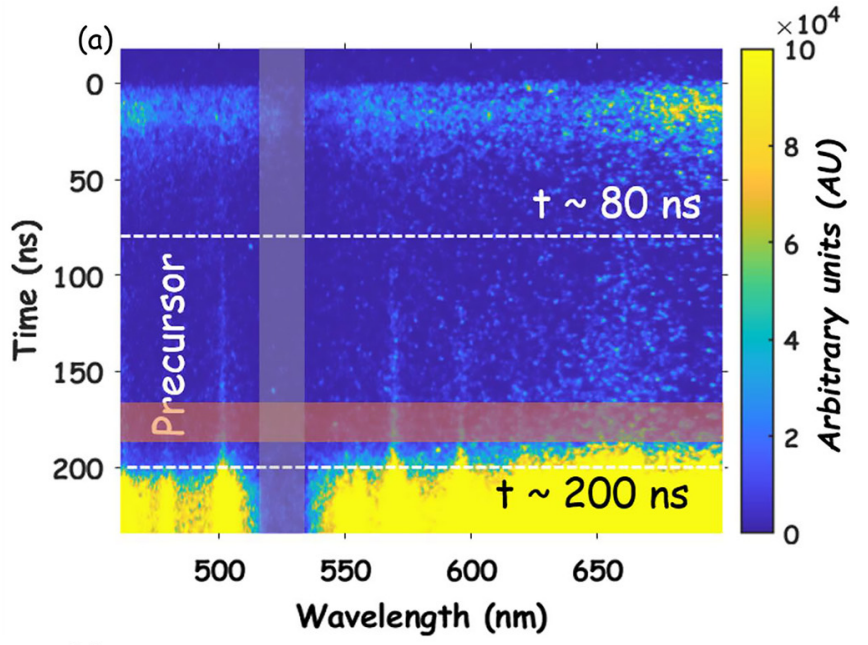

(b)

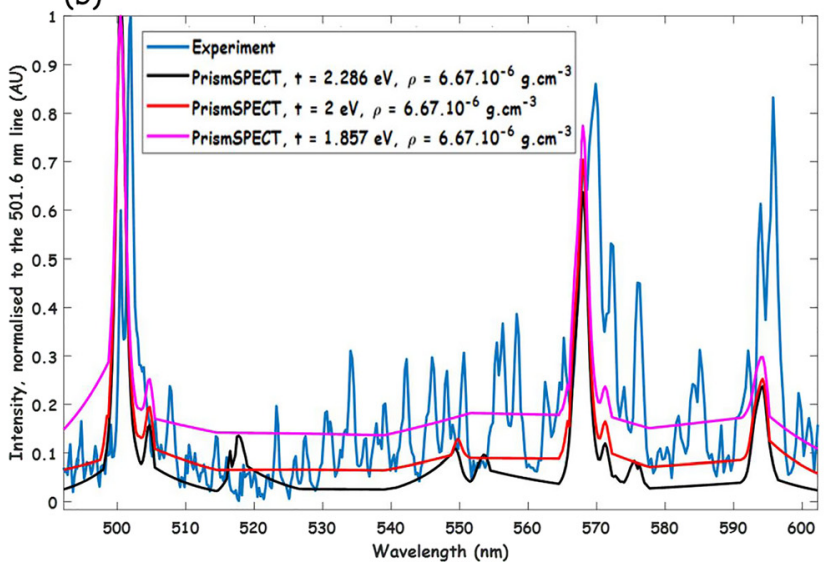

FIG. 5. (a) Zoom of Fig. 4 highlighting the apparition of lines prior to the arrival of the blast wave in $N_{2}$ gas at 5.7 mbar. The red lines correspond to the zone of the lineout of (b). (b) Lineout of (a) (red band in (a) where data are time-integrated over $10 \mathrm{~ns})$ and comparison with the simulation done using PrismSPECT and various ( $\rho$, T) conditions

between 200 and 270 ns. At $t=285$ ns, the FWHM becomes smaller $(\sim 2.4 \mathrm{~nm})$ and remains almost constant up to $t=385 \mathrm{~ns}$, which indicates a drop in density to $\sim 2-2.5 \times 10^{-5} \mathrm{~g} \mathrm{~cm}^{-3}$. It is interesting to compare these results with Fig. 3. The maximum electron density retrieved from interferometry is $\sim 9.5 \times 10^{18} \mathrm{~cm}^{-3}$ corresponding to $\sim 3.1 \times 10^{-5} \mathrm{~g} \mathrm{~cm}^{-3}$, i.e., in agreement with the density evaluated by time-resolved optical spectroscopy.

Figure 7 presents the comparison between PrismSPECT simulations at a fixed density $\left(3 \times 10^{-5} \mathrm{~g} \mathrm{~cm}^{-3}\right)$ and experimental data averaged from 230 to $240 \mathrm{~ns}$. The two green arrows on the upper right box of Fig. 7 show how the appearance/disappearance of lines allows to determine precisely the temperature. It is however important to note that these values are model dependent and should be taken with care. The best agreement, at $\mathrm{t} \sim 230-240 \mathrm{~ns}$, between the experimental spectrum and PrismSPECT is: $\rho=3 \times 10^{-5} \mathrm{~g} \mathrm{~cm}^{-3}$ and $T=3.9 \pm 0.1 \mathrm{eV}$. However, as can be seen in Fig. 7, a continuum exists, centered at $650 \mathrm{~nm}$. This feature incapacitates us to compare the lines ratio at 

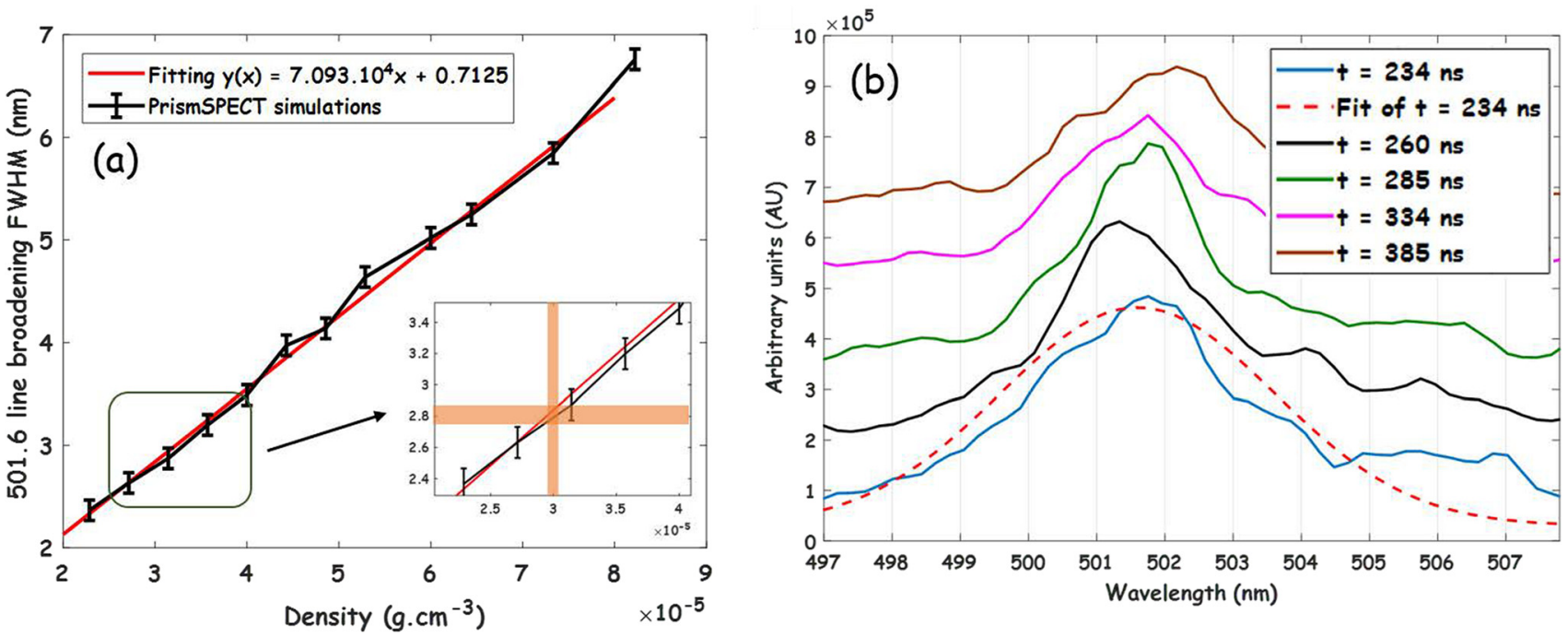

FIG. 6. (a) PrismSPECT simulations at a fixed temperature $(T=3.9 \mathrm{eV})$ which are used to calculate the FWHM of the $501.6 \mathrm{~nm}$ line, as a function of the density, using a $1 \mathrm{~nm}$ spectral resolution. The small box is a zoom around $2.4-4 \mathrm{~nm}$ of the $501.6 \mathrm{~nm}$ broadening. It corresponds to the conditions for $\mathrm{N}_{2}$ at $5.7 \mathrm{mbar}, 234 \mathrm{~ns}$ after the laser irradiation. (b) Experimental data for $\mathrm{N}_{2}$ at $5.7 \mathrm{mbar}$ of the $501.6 \mathrm{~nm}$ line (lineout of Fig. 4) for different times used to evaluate the FWHM. The red dashed line corresponds to a Gaussian fit of the data at $234 \mathrm{~ns}$. Its expression takes the form $f(x)=4.32510^{5} \exp \left(-((x-501.6) / 2.842)^{2}\right)$.

$650 \mathrm{~nm}$. Between 230 and $\sim 284 \mathrm{~ns}$, the temperature slightly drops to $3.8 \pm 0.2 \mathrm{eV}$ while the density remains constant $(\rho=3$ $\times 10^{-5} \mathrm{~g} \mathrm{~cm}^{-3}$ ). At $384 \mathrm{~ns}$, the temperature still drops to $3.4 \pm 0.2 \mathrm{eV}$ and the density to $\rho=2.5 \times 10^{-5} \mathrm{~g} \mathrm{~cm}^{-3}$. The physical conditions do not seem to evolve drastically after.

\section{E. Discussion}

Figure 8 summarizes the density and temperature profile measured by time resolved optical spectroscopy for $N_{2}$ at 5.7 mbar. The temperature profile [cf. Fig. 8(a)] shows the presence of a precursor ahead of the shock front. The temperature increase is due to photons emitted in the post-shock region, which then propagate in the downstream gas and preheat it. The radiative behavior of the BW has not been seen in analyzing its velocity [see Fig. 2(e)]. The radius of the BW evolves as $t^{2 / 5}$ although radiative losses exist. This should lower the power of $t$ as radiation removes energy from the shock. However, the uncertainties in determining precisely the radius of the BW, but also the laser energy fluctuations shot to shot, do not allow to conclude that the power of $t$ is lower than 2/5. The IR density evolution is shown in Fig. 8(b). A plateau is observed between $\sim 200$ and $300 \mathrm{~ns}$ and a slow rarefaction right after.

The Mach number in these conditions is relatively high, $\mathrm{M}>10$ (although the presence of high temperature gas $(\sim 2 \mathrm{eV})$ ahead of the shock (precursor) front should lower it) allowing the use of the strong shock approximation for the compression ratio $r=\rho_{1} / \rho_{2}$ $=(\gamma+1) /(\gamma-1)$ to determine the polytropic index $\gamma$, where $\rho_{1}$ is the downstream and $\rho_{2}$ is the upstream density. For $N_{2}$ at 5.7 mbar, $234 \mathrm{~ns}$ after the laser irradiation, this ratio $\mathrm{r}$ is not exactly equal to $4, \mathrm{r}$ $\sim 4.5 \pm 0.3$ leading to $\gamma \sim 1.57$. This has an important consequence; it helps to quantify the radiative nature of the shock (a fully radiative shock should tends toward 7 as $\gamma=4 / 3$ ). This shows as well the weak, but non negligible, influence of radiation on the shock evolution.
Instabilities $^{33-35}$ will not develop, as they grow only for gases with a polytropic index $\gamma<1.2$. $^{19}$

\section{DOUBLE BLAST WAVE MORPHOLOGY AND EVOLUTION}

The main objective of this paper is to determine the physical parameters of the interaction region (IR) formed by the collision of two Taylor-Sedov BWs and the plasma conditions. The evolution of the IR is illustrated in Fig. 9 for different gases and initial pressures. The dynamics of the zone is clearly different if one uses a $N_{2}$ or Ar gas [see Figs. 9(d) and 9(e)]. This is an important point as the nature of the gas changes the physical conditions and the dynamics of the IR. For example, the nature of the precursor ahead of a single blast wave front varies between $\mathrm{N}_{2}$ or Ar, as the mean free path for a given photon energy is different. This can modify the dynamics of the IR. For $\mathrm{N}_{2}$ at 5.7 mbar [see Fig. 9(a)], one can observe a narrow IR, $\sim 0.5 \mathrm{~mm}$, at early time $(\mathrm{t}=150 \mathrm{~ns})$ and stretch to $\sim 1.5 \mathrm{~mm}$ at $t=300 \mathrm{~ns}$. The amplitude of the signal increase indicates the presence of the steeper electron density gradient as well [see Fig. 9(e)]. For the Ar case at 2 mbar, one can observe a narrow IR at 150 and $200 \mathrm{~ns}$ about $0.5 \mathrm{~mm}$ in length. At later time $(t=300 \mathrm{~ns})$, the interaction region becomes hardly visible and spreads over a longer distance $(\sim 2 \mathrm{~mm})$. This means that the electron density gradient has become so long that it cannot be detected. In the following, we will concentrate our attention on the $\mathrm{N}_{2}$ case as it is easier for numerical perspective.

\section{A. Density and temperature of the interaction region}

The interferometric data does not allow a qualitative analysis. The only quantitative information retrieved about the IR is that, as we can follow the fringes (for the signal in the schlieren data, see Fig. 9), the density should not be above $\sim 1 \times 10^{20} \mathrm{~cm}^{-3}$. Figure 10 (a) presents the time resolved optical spectroscopy measurement made inside the 


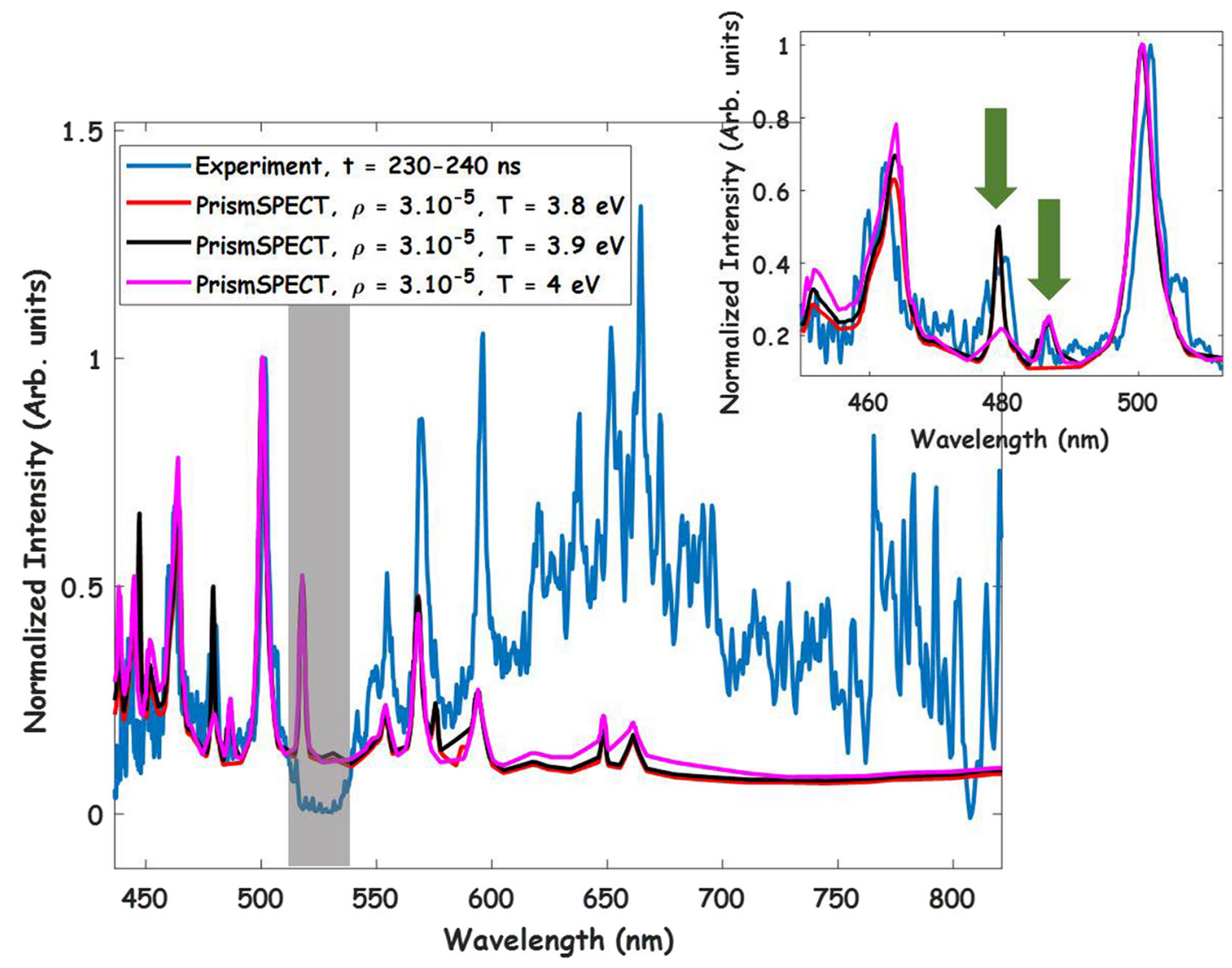

FIG. 7. Comparison between PrismSPECT simulations at a fixed density $\left(3 \times 10^{-5} \mathrm{~g} \mathrm{~cm}^{-3}\right)$ and experimental data averaged from 230 to $240 \mathrm{~ns}$ for $\mathrm{N}_{2}$ gas at $5.7 \mathrm{mbar}$. The upper right box corresponds to a zoom of the main image to show how one can determine precisely the temperature. The two green arrows show lines which appear (or disappears) when a slight variation of the temperature is applied. The gray band centered around $532 \mathrm{~nm}$ illustrates the notch filter that has been placed to remove the signal due to the main laser.
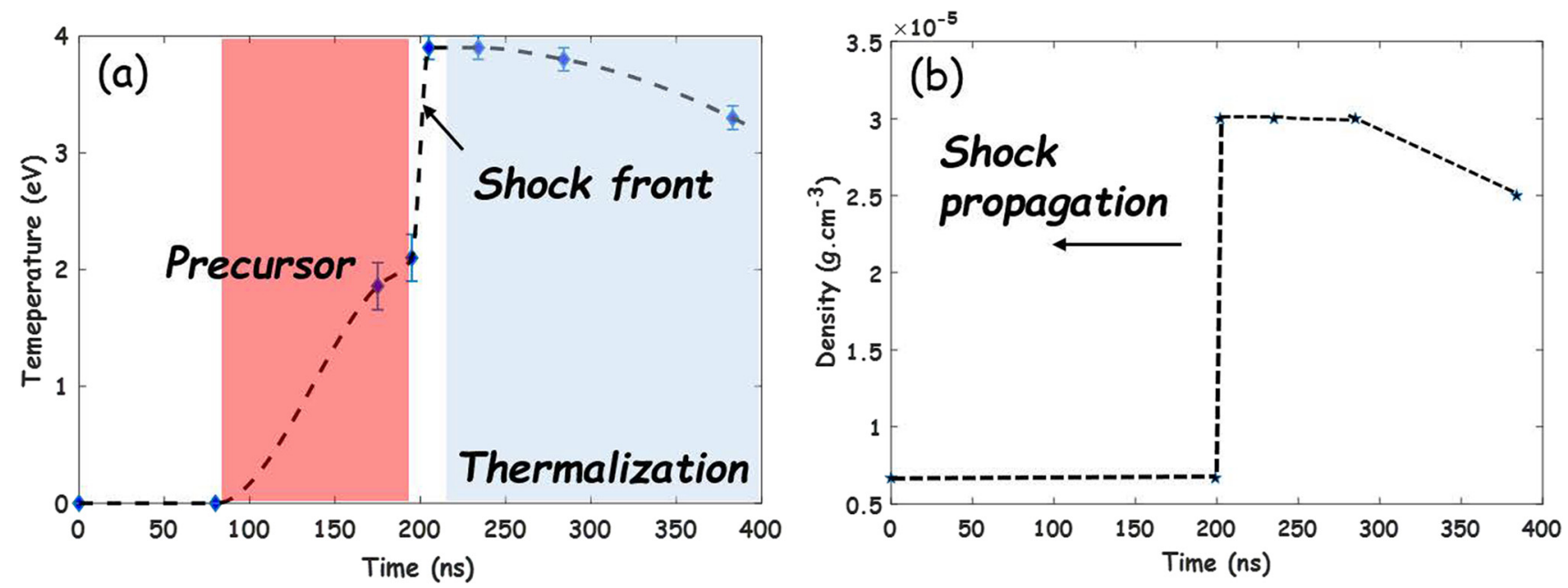

FIG. 8. (a) Temperature and (b) density of the blast wave measured by time resolved optical spectroscopy for $N_{2}$ at 5.7 mbar. 

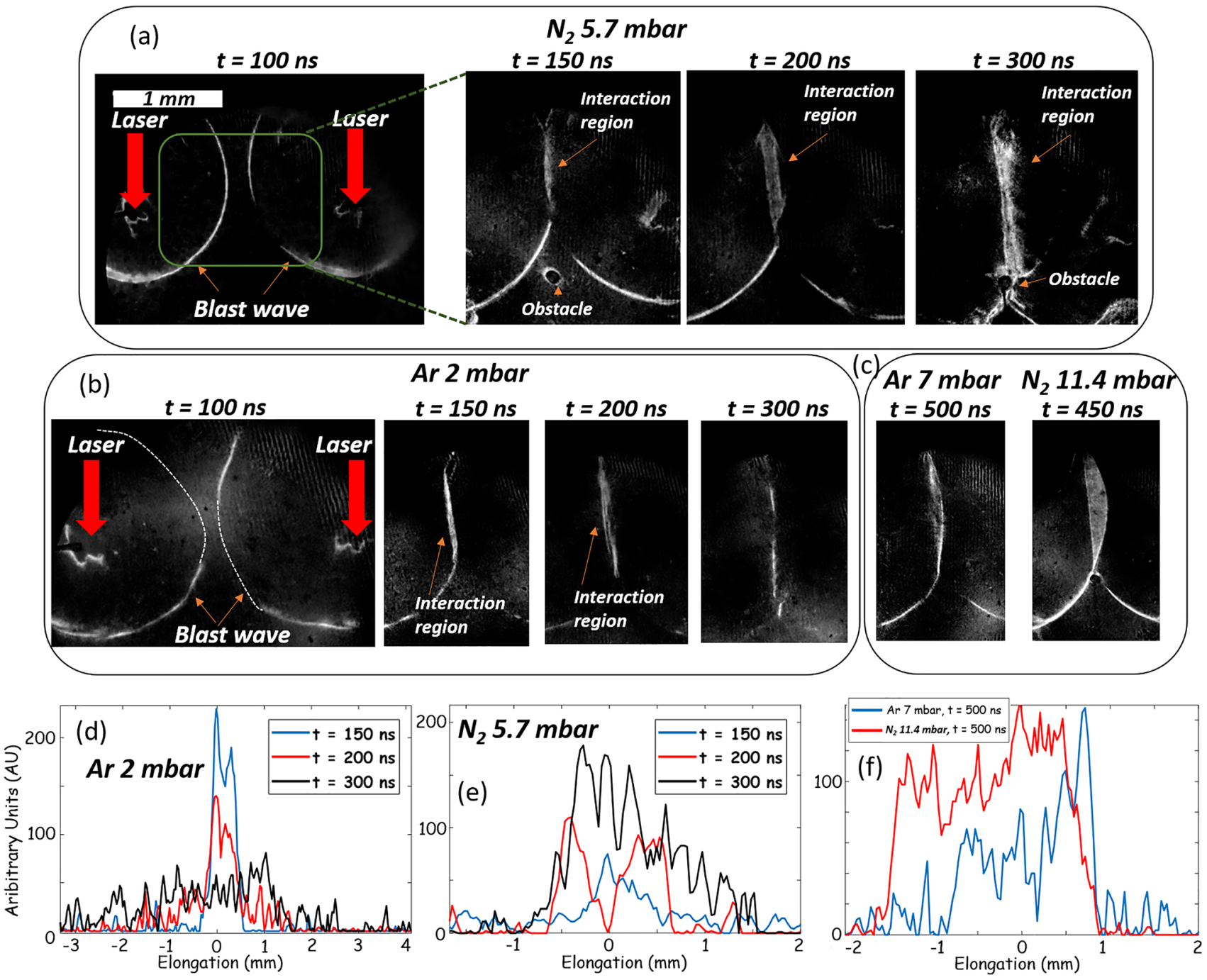

FIG. 9. Interaction of two Taylor-Sedov blast waves at different times, where $t=0$ corresponds to the laser arrival. (a) $N_{2}$ at 5.7 mbar and (b) Ar 2 mbar. (c) Comparison at the same mass density of the interaction region for $\operatorname{Ar} 7$ mbar and $N_{2} 11.4$ mbar. Lineout performed at the center of the interaction region (d) of (a), (e) of (b), and (f) of (c).

IR, in the same way as done for the single blast wave case (see Fig. 4). The precursor region is again measurable, from $\sim 80$ to $180 \mathrm{~ns}$ and has similar $(\rho, T)$ conditions $\left(\rho=6.67 \times 10^{-6} \mathrm{~g} \mathrm{~cm}^{-3}\right.$ (corresponding to the initial gas density) and $T=2.1 \pm 0.2 \mathrm{eV}$ ). The slight increase in the temperature compared with the precursor region of a single blast wave $(T=1.86 \pm 0.2 \mathrm{eV})$ is due to the interaction of both precursors. At about $180 \mathrm{~ns}$, the signal detected becomes stronger because the IR begins to enter in the probed zone of the diagnostic, and covers the entire region at $\sim 235 \mathrm{~ns}$. Thereafter, the progressive decrease in the signal is observed which indicates a cooling coupled with a decrease in the density.

A preliminary remark concerns the appearance and progressive disappearance of a line at $\sim 486 \mathrm{~nm}$. This line is not observed in the single blast wave case. According to PrismSPECT simulations, it appears, in the all levels model, at $T>4 \mathrm{eV}$. The same methodology as the single blast wave case (see Sec. III B) has been used to determine the density of the IR. At $\mathrm{t} \sim 244 \mathrm{~ns}$, the density is $\sim 5.3 \times 10^{-5} \mathrm{~g} \mathrm{~cm}^{-3}$ and remains almost constant up to $\sim 300 \mathrm{~ns}$. At $\mathrm{t} \sim 343 \mathrm{~ns}$, the density drops down to $\sim 4.2 \times 10^{-5} \mathrm{~g} \mathrm{~cm}^{-3}$ and becomes comparable to the single blast wave case at $t \sim 400 \mathrm{~ns}\left(\sim 3.4 \times 10^{-5} \mathrm{~g} \mathrm{~cm}^{-3}\right)$. The temperature of the IR can be determined precisely [see Fig. 10(b)]. It is of the order of $4.6 \pm 0.2 \mathrm{eV}$ at $\mathrm{t}=230-240 \mathrm{~ns}$. Thereafter, a slow but progressive cooling is observed, where $T=4.3 \pm 0.2 \mathrm{eV}$ at $290-300 \mathrm{~ns}$, drops to $4.0 \pm 0.2 \mathrm{eV}$ at $\mathrm{t}=343 \mathrm{~ns}$, to finally be $3.8 \pm 0.2 \mathrm{eV}$ at $400 \mathrm{~ns}$.

\section{B. Discussion}

\section{Density structure generation in the interaction region}

The experimental data (see Fig. 9) show no evidence of the formation of a Mach stem at the intersection point of the two BWs as in Ref. 27. The IR is inhomogeneous and strong electron density gradients are presents [see Figs. 9(d)-9(f)]. At $t=200 \mathrm{~ns}$, for $N_{2}$ at 5.7 

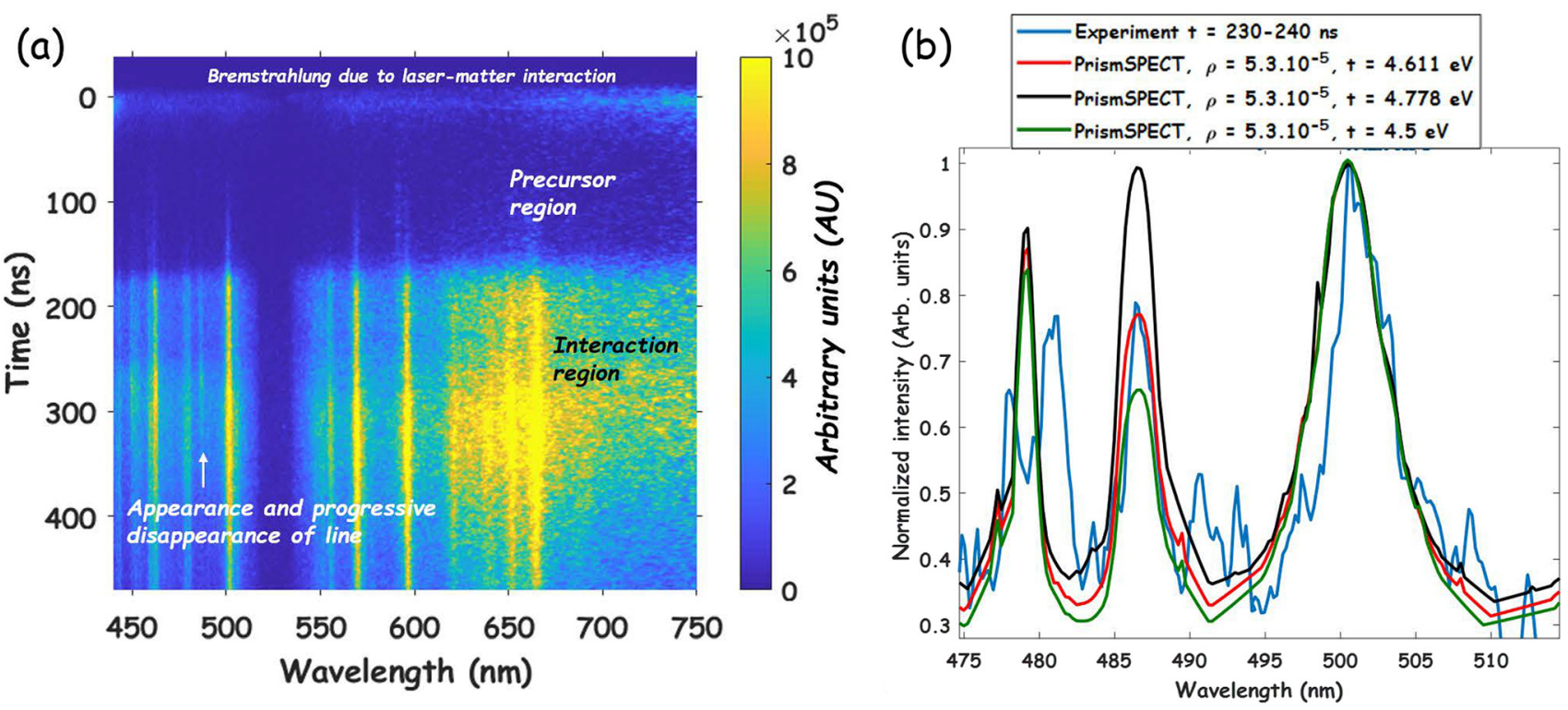

FIG. 10. (a) Typical results of time resolved optical spectroscopy with two blast waves propagating at 5.7 mbar of $N_{2}$. (b) Comparison between PrismSPECT simulations at a fixed density $5.3 \times 10^{-5} \mathrm{~g} \mathrm{~cm}^{-3}$ and experimental data averaged from 230 to $240 \mathrm{~ns}$ in the $475-500 \mathrm{~nm}$ range, allowing to determine precisely the temperature (according to the model used).

mbar [see Fig. 9(e)], one can also observe the formation of a cavity like structure (see Fig. 11). We attribute these strong electron density gradients to the formation of small scale vorticity produced when one BW encounters the internal structure of the second one. As a consequence, it is possible to generate a strong density structure at a long time after the beginning of the interaction of the two BW's due to vorticity generation. ${ }^{36}$ Figure 11 illustrates the time evolution of $\nabla n_{e}$ in the IR. At early time ( $t=150 \mathrm{~ns}$ ), the $\nabla n_{e}$ are not very pronounced while at later time, one can observe density structure generation. These structures could, in principle, generate B-fields by the Biermann Battery effect $\left(\nabla n_{e} \times \nabla T_{e}\right)$. Unfortunately, the spatial resolution of our diagnostic does not allow to determine accurately the spectrum of vorticity and its evolution during time.

\section{Reverse shock formation}

The formation of reverse shocks is also observed [see Figs. 11(b) and 11(e)]. They propagate in the post shock region of the other BWs. Up to $200 \mathrm{~ns}$, they are sharp and almost well defined [see Fig.11(b)], while at later time, there is no shock front but a smooth gradient [see Fig. 11(f)]. The mean velocity between 150 and $200 \mathrm{~ns}$ is about $15 \mathrm{~km} /$ s. It is however impossible to determine if it is in a Taylor-Sedov phase or not.

\section{Evolution of the interaction region: Cooling and rarefaction}

One can evaluate the compression as well as the increase in temperature inside the IR compared to the single BW case. The IR evolution of the thermodynamics conditions is summarized in Fig. 12. The maximum compression given by the ratio of the density in the IR over the density in the shell of a single blast wave is $\mathrm{C}=1.76$ for $N_{2}$ at 5.7 mbar and the temperature rises by $17 \%-20 \%$. It is interesting to note that the thermal equilibrium between electrons and ions is $<10 \mathrm{~ns}$ for the IR.

The time evolution of the density [see Fig. 12(a)] shows that the lateral expansion of the IR is constraint by the propagation of the two blast waves up to $\sim 300 \mathrm{~ns}$, i.e., during $\sim 70 \mathrm{~ns}$. At the same time, the temperature decreases slowly from 4.6 to $4.3 \mathrm{eV}$. After $300 \mathrm{~ns}$, the density and the temperature of the IR start to decrease to come back to a value similar to the single BW case at $\sim 400 \mathrm{~ns}$. The cooling function can be evaluated to be $\sim 5.4 \times 10^{-3} \mathrm{eV} / \mathrm{ns}$.

\section{ASTROPHYSICAL IMPLICATIONS}

The astrophysical system DEM L316 is composed of two distinct SNR's ${ }^{2}$ which had the particularity to explode at almost the same astrophysical time although they have been produced from different mechanisms (Supernovae of Type Ia for shell A and Type II for shell B). They are still in their Taylor-Sedov phase (determined by the physical properties of the hot gas). Preliminary analysis showed that the model derived $b^{2}{ }^{2}$ to explain astronomical observations reproduces most of the observed features (statically acceptable). However, the model did not succeed in reproducing the emission observed along the flattened part of the shell A toward shell B. Indeed, the brightest part of the inner ring of shell $\mathrm{A}$ is located near an emitting filament from shell $\mathrm{B}$. The main question raised from astronomical observations is: are they really interacting or just align in the line of sight of the observations? The different nature of the SNR progenitor raises some doubts that these SNRs interact. It should have been more understandable that both SNRs were formed in the same star formation region, having a comparable composition and thus explode after similar lifetimes. This is clearly not the case for DEM L316 ${ }^{2},{ }^{37}$ as one is probably a Type Ia SN and the second a Type II SNe, although the O/Fe and $\mathrm{Ne} / \mathrm{Fe}$ ratios for the Type II SNe being different from swept-up ISM 

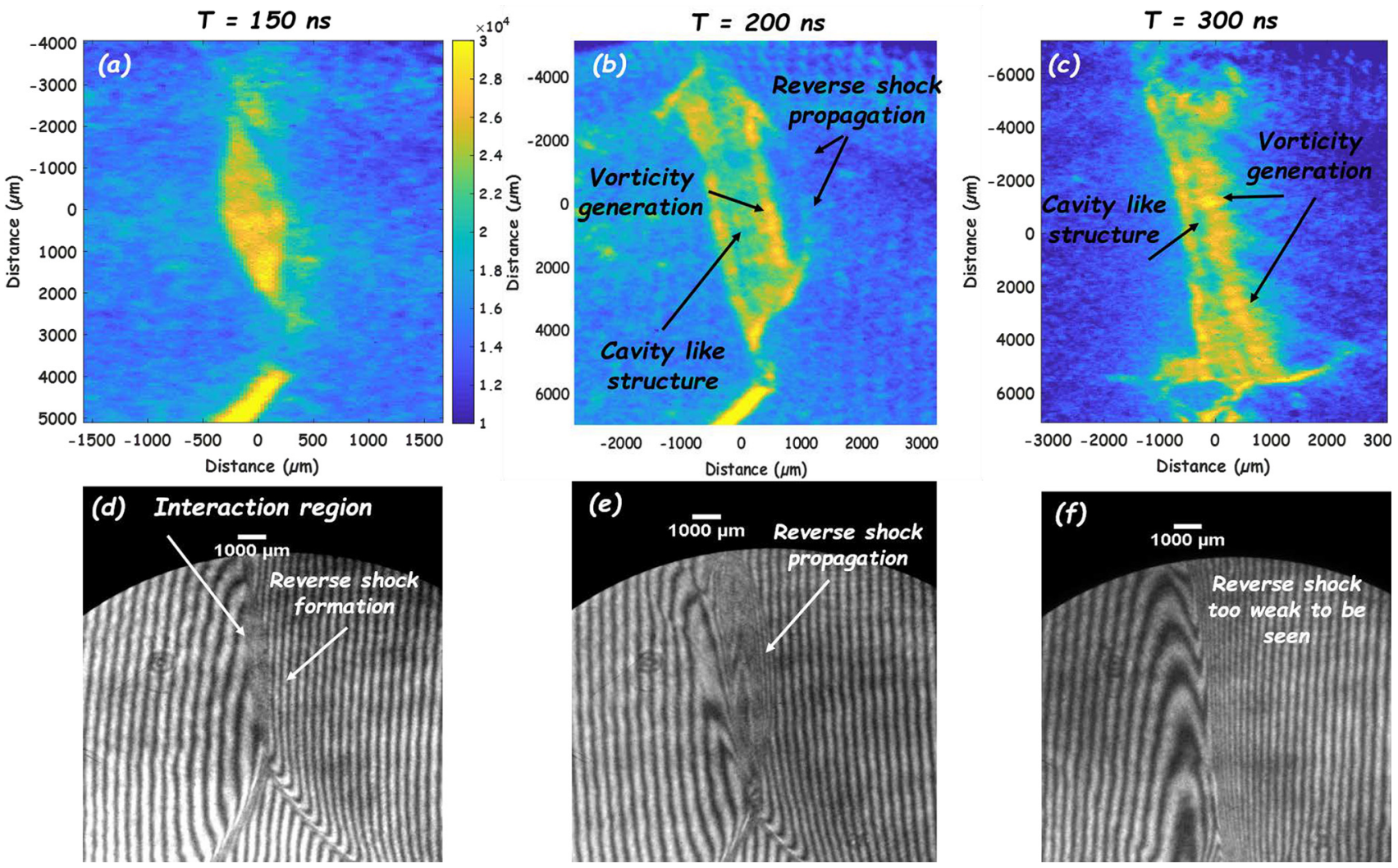

FIG. 11. Evolution of the interaction zone formed by the collision of two Taylor Sedov blast waves propagating in $\mathrm{N}_{2}$ at 5.7 mbar, seen by schlieren (up) and interferometry (bottom) data (a) and (d) at $\mathrm{t}=150 \mathrm{~ns}$, (b) and (e) at $\mathrm{t}=200 \mathrm{~ns}$, and (c) and (f) at $\mathrm{t}=300 \mathrm{~ns}$.

alone. This point is crucial as it strongly decreases the probability for the two SNRs to explode at the same astrophysical time.

What has been learned from the experiment which could help to answer to the SNRs interaction in DEM L316? The interaction region formed by the collision of two SNRs in their Taylor-Sedov phase is hotter than the shell of a single SNR. In experiment, we measure a maximum increase in the temperature of $\sim 18 \%$ in $N_{2}$ gas. These data can help to benchmark a numerical code. The next step is then to
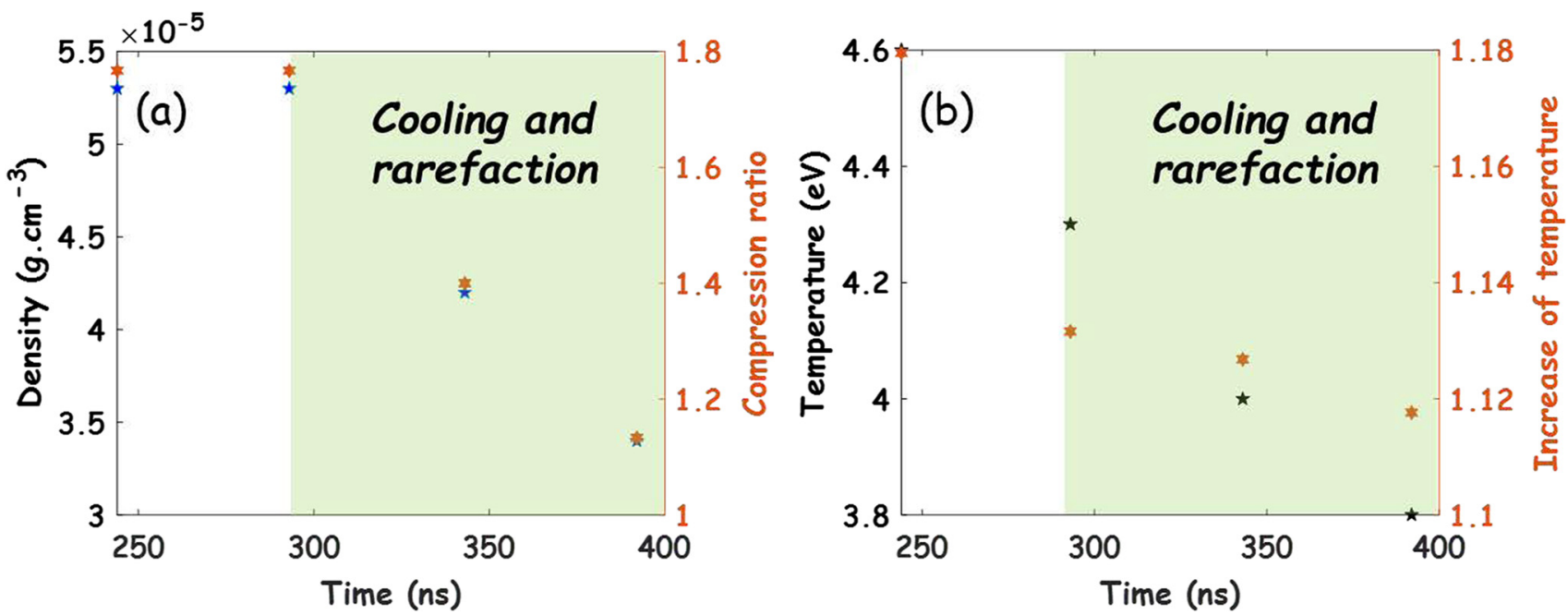

FIG. 12. Evolution of the thermodynamics conditions for the IR for $N_{2}$ at $5.7 \mathrm{mbar}$ (a) for density and (b) for temperature. 
perform atomic simulations to predict the expected emissions to compare them with the ones given in Ref. 2 and see if forbidden lines are generated. This is however beyond the scope of this paper.

In a more general sense, what has been learned from the experiment about the IR formed when two Taylor-Sedov blast waves collide? First, it has been shown (see Fig. 12) that vorticity generation occurs in the IR and leads to the formation of strong electron density gradients. Through the Biermann Battery effect, it is then possible to generate a small-scale magnetic field which could influence the polarization image of the Cygnus Loop. ${ }^{4}$ Second, we showed that the temperature of the IR remains high even at a long time after the beginning of the interaction. This can maintain hot interstellar gas even long time after the interaction. Third, the nature of the propagating medium (the ISM) is extremely important for the parameters of the IR. The SNRSNR interaction region will not evolve the same way (and have the same thermodynamic parameters) according to the background material present in the region. In that sense, specific scaling laws should be developed taking no conventional parameters for comparison (length of the precursor ahead of the shock front, compression ratio,...).

\section{CONCLUSION}

In conclusion, we have investigated experimentally the interaction region produced when two Taylor Sedov blast waves collide. We found: (1) density structure generation which could produce B-fields by the Biermann Battery effect. This could lead, in principle, to the formation of multiple small scale magnetized collisionless shocks in the IR and acceleration of charged particles. (2) A maximum compression ratio of $\sim 1.75$ and a $17 \%-20 \%$ increase in temperature in the IR for $\mathrm{N}_{2}$ at 5.7 mbar compared to the single BW case. The experimental results presented in this paper can help to constraint and validate hydrodynamic simulations. It represents a first step toward a better understanding of the physics of the SNR-SNR interaction. One interesting point is that the interaction region parameters depend on the nature of the gas. This is important when developing specific scaling laws as different parameters, will change according to the gas used, etc. which will change according to the gas used. It is then necessary to take this into account when comparing the laboratory experiment and the astrophysical system. In the future, it will be more relevant, from an astrophysical perspective, to perform large scale astrophysical simulations coupled to atomic modeling in order to predict the different emissions produced (x-ray and radio) and compare them to astronomical observations such as DEM L316. This could help to answer the questions of the interaction or not of the SNRs.

\section{ACKNOWLEDGMENTS}

The authors would like to thank the LULI staff for their contribution. This work has been done with the support of Investissements d'Avenir of LabEx PALM (Grant No. ANR-10LABX-0039-PALM). The JIHT RAS team works under the financial support from the Russian Foundation for Basic Research (Grant No. 18-29-21013). G.G. acknowledges support from the UK EPSRC (Grant Nos. EP/M022331/1 and EP/N014472/1). N.O. is grateful for the support under the International Joint Research Promotion program by Osaka University.

\section{REFERENCES}

1'B. W. Smith, Astrophys. J. 211, 404 (1977).

${ }^{2}$ R. M. Williams and Y. H. Chu, Astrophys. J. 635, 1077 (2005).

${ }^{3}$ P. Ambrocio-Cruz, M. Rosaldo, and E. de la Fuente, Rev. Mex. Astron. Astrofis. 42, 241-249 (2006).

${ }^{4}$ B. Uyaniker, W. Reich, A. Yar, R. Kothes, and E. Frst, Astron. Astrophys. 389, L61 (2002).

${ }^{\mathbf{5}}$ K. Yoshita, H. Tsunemi, E. Miyata, and K. Mori, Publ. Astron. Soc. Jpn. 53, 93 (2001).

${ }^{6}$ S. Broersen and J. Vink, Mon. Not. R. Astron. Soc. 446, 3885 (2015).

${ }^{7}$ R. M. Williams, Y. H. Chu, J. R. Dickel, R. Beyer, R. Petre, R. C. Smith, and D. K. Milne, Astrophys. J. 480, 618 (1997).

${ }^{8}$ D. Ryutov, R. P. Drake, J. Kane, E. Liang, B. A. Remington, and W. M. WoodVasey, Astrophys. J. 518, 821 (1999).

${ }^{9}$ P. Velarde, D. Garcia-Senz, E. Bravo, F. Ogando, A. Relano, C. Garcia, and E. Oliva, Phys. Plasmas. 13, 092901 (2006).

${ }^{10}$ E. Falize, C. Michault, and S. Bouquet, Astrophys. J. 730, 96 (2011).

${ }^{17}$ T. Ditmire, R. A. Smith, J. W. G. Tish, and M. H. R. Hutchinson, Phys. Rev. Lett. 78, 3121 (1997).

${ }^{12}$ K. Shigemori, T. Ditmire, B. A. Remington, V. Yanovsky, D. Ryutov, K. G. Estabrook, M. J. Edwards, A. J. MacKinnon, A. M. Rubenchik, K. A. Keilty, and E. Liang, Astrophys. J. 533, L159 (2000).

${ }^{13}$ T. Ditmire, K. Shigemori, B. A. Remington, K. Estabrook, and R. A. Smith, Astrophys. J. Suppl. Ser. 127, 299 (2000).

${ }^{14}$ M. J. Edwards, A. J. MacKinnon, J. Zweiback, K. Shigemori, D. Ryutov, A. M. Rubenchik, K. A. Keilty, E. Liang, B. A. Remington, and T. Ditmire, Phys. Rev. Lett. 87, 085004 (2001).

${ }^{15}$ A. S. Moore, J. Lazarus, M. Hohenberger, J. S. Robinson, E. T. Gumbrell, M. Dunne, and R. A. Smith, Astrophys. Space Sci. 307, 139 (2007).

${ }^{16}$ A. S. Moore, E. T. Grumbell, J. Lazarus, M. Hohenberger, J. S. Robinson, R. A. Smith, T. J. A. Plant, D. R. Symes, and M. Dunne, Phys. Rev. Lett. 100, 055001 (2008).

${ }^{17}$ D. R. Symes, M. Hohenberger, J. Lazarus, J. Osterhoff, A. S. Moore, R. R. Faustlin, A. D. Edens, H. W. Doyle, R. E. Carley, A. Marocchino, J. P. Chittenden, A. C. Bernstein, E. T. Grumbell, M. Dunne, and R. A. Smith, High Energy Density Phys. 6, 274 (2010).

${ }^{18}$ M. Hohenberger, D. R. Symes, J. Lazarus, H. W. Doyle, R. E. Carley, A. S. Moore, E. T. Grumbell, M. M. Notley, R. J. Clarke, M. Dunne, and R. A. Smith, Phys. Rev. Lett. 105, 205003 (2010).

${ }^{19}$ J. Grun, J. Stamper, C. Manka, J. Resnick, R. Burris, J. Carwford, and B. H. Ripin, Phys. Rev. Lett. 66, 2738 (1991).

${ }^{20}$ A. D. Edens, T. Ditmire, J. F. Hansen, M. J. Edwards, R. G. Adams, P. Rambo, L. Ruggles, I. C. Smith, and J. L. Porter, Phys. Plasmas 11, 4968 (2004).

${ }^{21}$ A. D. Edens, T. Ditmire, J. F. Hansen, M. J. Edwards, R. G. Adams, P. Rambo, L. Ruggles, I. C. Smith, and J. L. Porter, Phys. Rev. Lett. 95, 244503 (2005).

${ }^{22}$ J. F. Hansen, M. J. Edwards, D. H. Froula, G. Gregori, A. D. Edens, and T. Ditmire, Phys. Plasmas 13, 022105 (2006).

${ }^{23}$ A. D. Edens, R. G. Adams, P. Rambo, L. Ruggles, I. C. Smith, J. L. Porter, and T. Ditmire, Phys. Plasmas 17, 112104 (2010).

${ }^{24}$ R. R. Elton, D.-M. Billings, C. K. Manka, H. R. Griem, J. Grun, B. H. Ripin, and J. Resnick, Phys. Rev. E 49, 1512 (1994).

${ }^{25}$ R. A. Bosch, R. L. Berger, B. H. Failor, N. D. Delamater, G. Charatis, and R. L. Kauffman, Phys. Fluid. B 4, 979 (1992).

${ }^{26}$ R. A. Smith, J. Lazarus, M. Hohenberger, A. S. Moore, J. S. Robinson, E. T. Grumbell, and M. Dunne, Astrophys. Space Sci. 307, 131 (2007).

${ }^{27}$ R. A. Smith, J. Lazarus, M. Hohenberger, A. Marocchino, J. S. Robinson, J. P. Chittenden, A. S. Moore, E. T. Grumbell, and M. Dunne, Plasma Phys. Controlled Fusion 49, B117 (2007).

${ }^{28}$ F. Suzuki-Vidal, T. Clayson, C. Stehlé et al., Phys. Rev. Lett. 119, 055001 (2017).

${ }^{29}$ K. Bockasten, J. Opt. Soc. Am. 51, 943 (1961).

30 J. J. MacFarlane, I. E. Golovkin, P. Wang, P. R. Woodruff, and N. A. Pereyra, High Energy Density Phys. 3, 181 (2007).

${ }^{31}$ Y. B. Zel'dovich and Y. P. Raizer, Physics of Shock Waves and HighTemperature Hydrodynamic Phenomena (Academic Press, New York, 1967). 
${ }^{32}$ T. Michel, E. Falize, B. Albertazzi, G. Rigon, Y. Sakawa, T. Sano, H. Shimogawara, R. Kumar, T. Morita, C. Michault, A. Casner, P. Barroso, P. Mabey, Y. Kuramitsu, S. Laffite, L. V. B. Som, G. Gregori, R. Kodama, N. Ozaki, P. Tzeferacos, D. Lamb, and M. Koenig, High Power Laser Sci. Eng. 6 , e30 (2018).

${ }^{33}$ D. Ryu and E. T. Vishniac, Astrophys. J. 368, 411 (1991)
${ }^{34}$ D. Ryu and E. T. Vishniac, Astrophys. J. 313, 820 (1987).

${ }^{35}$ E. T. Vishniac, Astrophys. J. 274, 152 (1983).

${ }^{36}$ A. P. L. Robinson, H. Schmitz, T. E. Fox, J. Pasley, and D. R. Symes, High Energy Density Phys. 14, 6 (2015).

${ }^{37}$ M. Nishiuchi, J. Yokogawa, K. Koyama, and J. P. Hughes, Publ. Astron. Soc. Jpn. 53, 99 (2001). 